\title{
RUPTURE SOLUTIONS OF AN ELLIPTIC EQUATION WITH A SINGULAR NONLINEARITY
}

\author{
ZONGMING GUO AND JUNCHENG WEI
}

\begin{abstract}
We construct infinitely many non-radial rupture so-
\end{abstract} lutions of the equation

with

$$
\Delta u=\frac{1}{u^{p}} \text { in } \mathbb{R}^{N} \backslash\{0\}, u(0)=0, \quad N \geq 3
$$

$$
p>p_{c}(N-1):=\frac{N-1-2 \sqrt{N-2}}{2 \sqrt{N-2}-(N-5)} .
$$

\section{INTRODUCTION}

Of concern is the following nonlinear elliptic equation with negative exponent

$$
\Delta u-u^{-p}=0 \text { in } \mathbb{R}^{N}
$$

where $p>0$ and $N \geq 2$.

Equation (1.1) arises in the study of steady states of thin films of viscous fluids and in the modeling of electrostatic micro-electromechanical systems (MEMS). It has attracted extensive studies in recent years, see, for example, Davila-Ponce [3], Esposito-Ghoussoub-Guo ([6], [7]), Esposito [5], Ghoussoub-Guo [8], Guo-Ye-Zhou [10], Guo-Wei [11]-[13], Jiang-Ni $[15]$ and the references therein.

When $p>0$, the structure of positive radial solutions of (1.1) has been studied by Guo, Guo and Li [9], Guo and Wei [11]. It is shown that for any $a>0$, equation (1.1) admits a unique positive radial solution $u=u(r)$ such that $u(0)=a$ and $u(r) \rightarrow+\infty$ as $r \rightarrow+\infty$.

1991 Mathematics Subject Classification. 35J25, 35J20, 35B33, 35B40.

Key words and phrases. Nonradial rupture solutions, singular nonlinearity, asymptotic analysis, gluing.

The research of the first author is supported by NSFC (11171092) and Innovation Scientists and Technicians Troop Construction Projects of Henan Province (114200510011). The research of the second author is supported by an Earmarked Grant from RGC of Hong Kong and Oversea Joint Grant of NSFC.. 
The solution $u$ satisfies $u^{\prime}(r)>0$ for all $r>0$ and

$$
\lim _{r \rightarrow \infty} r^{-\frac{2}{p+1}} u(r)=\left[\frac{2}{p+1}\left(N-2+\frac{2}{p+1}\right)\right]^{-\frac{1}{p+1}}\left(:=\beta^{-\frac{1}{p+1}}\right) .
$$

Moreover, if $2 \leq N \leq 9$ and

$$
p>p_{c}(N)
$$

then $u(r)-\beta^{-\frac{1}{p+1}} r^{\frac{2}{p+1}}$ changes sign infinitely many times. If $N \geq 3$ and $0<p \leq p_{c}(N)$, then $u(r)>\beta^{-\frac{1}{p+1}} r^{\frac{2}{p+1}}$ for all $r>0$ and the solutions are strictly ordered with respect to the initial value $a=u(0)$. Here $p_{c}(M)$ ( $M$ is an integer) is the Joseph-Lundgren exponent [14]:

$$
p_{c}(M):= \begin{cases}P(M), & \text { if } 2 \leq M \leq 9 \\ \infty, & \text { if } M \geq 10\end{cases}
$$

where

$$
P(M)=\frac{M-2 \sqrt{M-1}}{2 \sqrt{M-1}-(M-4)} .
$$

Note that $P(M)$ is an increasing function of $M$ and $p_{c}(2)=0$.

When $p>0$, it is still open if all positive solutions are radially symmetric about some point. It is shown in [12] that if $u \in C^{2}\left(\mathbb{R}^{N}\right)$ is a positive solution of (1.1), and

$$
\lim _{|x| \rightarrow \infty}|x|^{-\frac{2}{p+1}} u(x)=\beta^{-\frac{1}{p+1}},
$$

then $u$ is radially symmetric about some point $x_{0} \in \mathbb{R}^{N}$. Some qualitative properties of entire solutions of (1.1) are studied in [16]. Solutions of (1.1) are also classified by using the Morse index theory. Du and Guo [4] showed that (1.1) does not admit any positive regular solution that has finite Morse index provided that $p>p_{c}(N)$.

Now we turn to rupture solutions to (1.1). The rupture set of (1.1) consists of the points where $u$ vanishes. We consider the simplest case where the rupture is the origin. Thus we consider the following problem

$$
\Delta u-u^{-p}=0, \quad u>0, \quad \text { in } \mathbb{R}^{N} \backslash\{0\}, \quad u(0)=0 .
$$

When $p>0$, the radial rupture solution to (1.2) takes the form

$$
U(x):=U(|x|)=\left[\frac{2}{p+1}\left(N-2+\frac{2}{p+1}\right)\right]^{-\frac{1}{p+1}}|x|^{\frac{2}{p+1}} .
$$

A basic question we will address in this paper is whether or not all rupture solutions to (1.2) are radially symmetric (and hence are of the 
form (1.3)). Note that when $N=2$ and $p=3$, there is a family of non-radial rupture solution

$$
\sqrt{2|x|}\left(\epsilon\left(\cos \frac{\theta}{2}\right)^{2}+\epsilon^{-1}\left(\sin \frac{\theta}{2}\right)^{2}\right)^{\frac{1}{2}} .
$$

Besides (1.4), we are not aware of any non-radial rupture solutions to (1.2). The purpose of this paper is to construct infinitely many positive non-radial rupture solutions of (1.2) provided

$$
p>p_{c}(N-1) \text {. }
$$

This gives an negative answer to the above question. Note that $p_{c}(M)$ is an increasing function of $M$. Then $p_{c}(N)>p_{c}(N-1)$. This provides new information on the case $p \leq p_{c}(N)$. Note also that

$$
\left(M-2+\frac{4}{p+1}\right)^{2}-8\left(M-2+\frac{2}{p+1}\right)<0, \text { for } p>p_{c}(M) .
$$

Our main result can be stated as follows.

Theorem 1.1. Assume that

$$
p>p_{c}(N-1) .
$$

Then there exist infinitely many non-radial rupture solutions to (1.2).

An interesting consequence of Theorem 1.1 is the following.

Corollary 1.2. For each $p>0$, there are infinitely many non-radial rupture solutions to

$$
\Delta u=u^{-p} \text { in } \mathbb{R}^{3}, \quad u(0)=0 .
$$

The idea of proving Theorem 1.1 is simple: we look for separable solutions

$$
u(x)=r^{\frac{2}{p+1}} w(\omega)
$$

where $w$ is a solution of the equation

$$
\Delta_{S^{N-1}} w+\beta w-w^{-p}=0,
$$

with

$$
\beta=\frac{2}{p+1}\left(N-2+\frac{2}{p+1}\right) .
$$

An obvious solution to $(1.8)$ is $w(\omega) \equiv \beta^{-\frac{1}{p+1}}$ which provides a radial rupture solution of (1.1) as given in (1.3).

To construct positive non-radial rupture solutions of (1.1), we need to find positive non-constant solutions $w(\omega)$ of (1.8). In this paper, we will construct infinitely many positive radially symmetric solutions of (1.8), i.e., solutions that only depend on the geodesic distance $\theta \in[0, \pi)$. 
In this case, (1.8) can be written in a more convenient form (with $x=\cos \theta)$, namely

$\left\{\begin{array}{l}\left(1-x^{2}\right)^{-\frac{N-3}{2}}\left(\left(1-x^{2}\right)^{\frac{N-1}{2}} w_{x}\right)_{x}+\beta w-w^{-p}=0, \quad w(x)>0,-1<x<1 \\ w^{\prime}(1) \text { exists. }\end{array}\right.$

Furthermore we only consider the simple case $w(-x)=w(x)$ for $x \in(0,1)$. Thus $w^{\prime}(0)=0$. Then $w(x):=w(\theta)$ with $w(\theta)=w(\pi-\theta)$ for $0<\theta \leq \pi / 2$ satisfies the problem

$$
\left\{\begin{array}{l}
\frac{1}{\sin ^{N-2} \theta} \frac{d}{d \theta}\left(\sin ^{N-2} \theta \frac{d w}{d \theta}(\theta)\right)+\beta w(\theta)-w^{-p}(\theta)=0, \quad w(\theta)>0, \quad 0<\theta<\frac{\pi}{2} \\
w_{\theta}^{\prime}(0) \text { exists, } \quad w_{\theta}^{\prime}\left(\frac{\pi}{2}\right)=0 .
\end{array}\right.
$$

Even though problem (1.10) is just an ODE, it is clearly supercritical. Neither the variational method nor the sub-super solution method nor the bifurcation method works. An important observation is that

$$
w_{*}(\theta)=A_{p}[\sin \theta]^{\frac{2}{p+1}}, \quad \theta \in\left(0, \frac{\pi}{2}\right], \quad A_{p}^{-(p+1)}=\frac{2}{p+1}\left[N-3+\frac{2}{p+1}\right]
$$

is a rupture solution of (1.10) with two ruptures: $\theta=0$ and $\theta=\pi$. We will construct the inner and outer solutions of (1.10) and then glue them to be solutions of (1.10). Such arguments have been used in [1] for the supercritical problem $\Delta u+\lambda u+u^{p}=0$ in a unit ball in $\mathbb{R}^{3}$ with $p>5$. This idea was also used in [2] for constructing non-radial singular solutions to Lane-Emden equation $\Delta u+u^{p}=0$.

The non-radial rupture solutions to (1.1) may serve as good asymptotics for non-radial entire solutions to (1.1). We conjecture that for each of the non-radial singular solutions $r^{\frac{2}{p+1}} w(\theta)$ constructed in Theorem 1.1 there exists an entire positive solution $u$ to (1.1) such that

$$
u(x)-|x|^{\frac{2}{p+1}} w(\theta)=o\left(|x|^{\frac{2}{p+1}}\right), \text { for }|x| \gg 1 .
$$

This paper is organized as follows: in Section 2, we study an initial value problem and study the asymptotic behavior of the inner solution when the initial value turns to 0 . In Section 3, we study the outer problem. Namely we solve the problem (1.10) from $\theta=\frac{\pi}{2}$. The asymptotic behavior of the outer problem will be analyzed near the origin. Finally in Section 4, we do a matching of inner and outer solution, thereby proving Theorem 1.1 . 


\section{INNER SOLUTIONS}

In this section we study solutions $w(\theta)$ of (1.10) with small initial values $w(0)=Q \ll 1$ and analyze their behaviors near $\theta=0$. Since $Q \ll 1$, we set $Q=\epsilon^{\frac{2}{p+1}}\left(:=\epsilon^{\alpha}\right)$ with $\epsilon$ sufficiently small. In the following we denote $\alpha=2 /(p+1)$.

Let $w(\theta)=\epsilon^{\alpha} v\left(\frac{\theta}{\epsilon}\right)$. Then we see that $v(0)=1$ and $v(r)(r=\theta / \epsilon)$ satisfies

$$
v_{r r}+(N-2) \epsilon \cot (\epsilon r) v_{r}+\beta \epsilon^{2} v-v^{-p}=0, \quad v(0)=1 .
$$

Observe that for $\epsilon>0$ sufficiently small,

$$
\cot (\epsilon r)=\frac{\cos (\epsilon r)}{\sin (\epsilon r)}=\frac{1}{\epsilon r}-\frac{1}{3}(\epsilon r)+\sum_{k=1}^{\infty} \ell_{k}(\epsilon r)^{2 k+1} .
$$

Thus,

$$
v_{r r}+\frac{N-2}{r} v_{r}-\frac{(N-2)}{3}\left(\epsilon^{2} r\right) v_{r}+\left(\sum_{k=1}^{\infty}(N-2) \ell_{k} \epsilon^{2(k+1)} r^{2 k+1}\right) v_{r}+\beta \epsilon^{2} v-v^{-p}=0, \quad v(0)=1 .
$$

When $\epsilon=0$, we obtain the first approximation of (2.2) which is the radial solution $v_{0}(r)$ of the problem

$$
\Delta v-v^{-p}=0 \quad \text { in } \mathbb{R}^{N-1}, \quad v(0)=1 .
$$

The asymptotic behavior of $v_{0}$ is given in the following lemma.

Lemma 2.1. For $p>p_{c}(N-1)$, there exist constants $a_{0}, b_{0}$ and $R_{0} \gg 1$ such that for $r \geq R_{0}$ the unique positive solution $v_{0}(r)$ of (2.3) satisfies

$$
v_{0}(r)=A_{p} r^{\alpha}+\frac{a_{0} \cos (\omega \ln r)+b_{0} \sin (\omega \ln r)}{r^{\frac{N-3}{2}}}+O\left(r^{-\left(N-3+\frac{2}{p+1}\right)}\right),
$$

where

$$
\begin{gathered}
A_{p}^{-(p+1)}=\frac{2}{p+1}\left[N-3+\frac{2}{p+1}\right] \\
\omega=\frac{1}{2} \sqrt{8\left(N-3+\frac{2}{p+1}\right)-\left(N-3+\frac{4}{p+1}\right)^{2}} .
\end{gathered}
$$

Proof. Note that

$$
8\left(N-3+\frac{2}{p+1}\right)-\left(N-3+\frac{4}{p+1}\right)^{2}>0, \text { for } p>p_{c}(N-1) .
$$

The existence and uniqueness of $v_{0}(r)$ can found in [9] and [11]. It is also known from [9] and [11] that

$$
\lim _{r \rightarrow+\infty} r^{-\alpha} v_{0}(r)=A_{p} .
$$


To obtain the next order expansion, we use the Emden-Fowler transformation:

$$
V(t)=r^{-\alpha} v_{0}(r)-A_{p}, \quad t=\ln r .
$$

It is easy to see that $V(t)$ satisfies the equation

$V_{t t}+(N-3+2 \alpha) V_{t}+2(N-3+\alpha) V-g(V)=0, \quad$ for $t \geq T=\ln R, \quad R>10$, where $g(\rho)=\left(\rho+A_{p}\right)^{-p}-A_{p}^{-p}+p A_{p}^{-(p+1)} \rho$ satisfies

$$
g(\rho)=\frac{p(p+1)}{2} A_{p}^{-(p+2)} \rho^{2}+O\left(\rho^{3}\right) \text { for } \rho \text { near } 0 .
$$

By the standard argument of variation of constants we obtain the following integral equation

$$
V(t)=e^{\sigma t}[a \cos \omega t+b \sin \omega t]-\frac{1}{\omega} \int_{T}^{t} e^{\sigma\left(t-t^{\prime}\right)} \sin \omega\left(t-t^{\prime}\right) g\left(V\left(t^{\prime}\right)\right) d t^{\prime},
$$

where $\sigma=-\frac{1}{2}(N-3+2 \alpha), \omega$ is given in (2.5). Note that $g(\rho)=O\left(\rho^{2}\right)$ for $\rho$ sufficiently small.

Set $\tilde{V}(t)=e^{-\sigma t} V(t)$. Then $\tilde{V}(t)$ satisfies the integral equation

$$
\tilde{V}(t):=\mathcal{N} \tilde{V}(t)=C \sin (\omega t+D)-\frac{1}{\omega} \int_{T_{0}}^{t} e^{-\sigma t^{\prime}} \sin \omega\left(t-t^{\prime}\right) g\left(e^{\sigma t^{\prime}} \tilde{V}\left(t^{\prime}\right)\right) d t^{\prime},
$$

where $C=\sqrt{a^{2}+b^{2}}, \sin D=\frac{a}{C}, \cos D=\frac{b}{C}$. We take $t$ in the range $T_{0} \leq t<\infty$, where $T_{0}=\ln R_{0}$ is suitably large, and consider $\mathcal{N} \tilde{V}$ as a map from $C\left[T_{0}, \infty\right)$ into itself. We claim that, for each $C>0$ and suitable $T_{0}$, the operator $\mathcal{N} \tilde{V}$ maps the set

$$
\mathcal{B}=\left\{\tilde{V} \in C\left[T_{0}, \infty\right):\|z\|_{0}=\sup _{T_{0}<t<\infty}|\tilde{V}(t)| \leq 2 C, \quad C>0\right\}
$$

into itself, and is a contraction mapping on $\mathcal{B}$. Indeed, if $\|\tilde{V}\|_{0}<2 C$, then

and

$$
\left|g\left(e^{\sigma t} \tilde{V}(t)\right)\right|=e^{2 \sigma t} O(1)
$$

$$
\|\mathcal{N} \tilde{V}-C \sin (\omega t+D)\|_{0} \leq C^{\prime} e^{\sigma T_{0}}
$$

where $C^{\prime}>0$ only depends on $C, N, p$. Note that $\sigma<0$ and $\left\|e^{\sigma t} \tilde{V}(t)\right\|_{0}$ is sufficiently small for $\tilde{V} \in \mathcal{B}$ for $T_{0}$ suitably large. Thus, if we choose $T_{0}>1$ suitably large, we see that $\|\mathcal{N} \tilde{V}-C \sin (\omega t+D)\|_{0}<C$. A similar calculation shows that

$$
\left\|\mathcal{N} \tilde{V}_{1}-\mathcal{N} \tilde{V}_{2}\right\|_{0} \leq e^{\sigma T_{0}}\left\|\tilde{V}_{1}-\tilde{V}_{2}\right\|_{0}
$$

Hence it is possible for each value of $C$ to choose $T_{0}$ so that $\mathcal{N}$ is a contraction mapping of $\mathcal{B}$ to itself. Thus, we define $\tilde{V}_{0}=C \sin (\omega t+D)$ 
and the iteration $\tilde{V}_{n+1}=\mathcal{N} \tilde{V}_{n}$ for $n \geq 0$. The contraction mapping theorem then ensures that this iteration converges to the unique solution $\tilde{V}_{*}(t)$ of $(2.7)$ in $\mathcal{B}$. Note that

$$
\left|\frac{1}{\omega} \int_{T_{0}}^{t} e^{-\sigma t^{\prime}} \sin \omega\left(t-t^{\prime}\right) g\left(e^{\sigma t^{\prime}} \tilde{V}_{*}\left(t^{\prime}\right)\right) d t^{\prime}\right|=O\left(e^{\sigma t}\right) .
$$

Then

$$
V_{*}(t)=e^{\sigma t} \tilde{V}_{*}(t)=C_{0} e^{\sigma t} \sin \left(\omega t+D_{0}\right)+O\left(e^{2 \sigma t}\right) \text { for } t \in\left(T_{0}, \infty\right) .
$$

This implies that for $r \in\left[R_{0}, \infty\right)$,

$$
v_{0}(r)=A_{p} r^{\alpha}+r^{-\frac{N-3}{2}}\left[a_{0} \cos (\omega \ln r)+b_{0} \sin (\omega \ln r)\right]+O\left(r^{-\left(N-3+\frac{2}{p+1}\right)}\right)
$$

and completes the proof of this lemma.

Lemma 2.2. Let $p$ satisfy the conditions of Lemma 2.1 and $v_{1}(r)$ be the unique solution of the problem

$$
\left\{\begin{array}{l}
v_{1}^{\prime \prime}(r)+\frac{N-2}{r} v_{1}^{\prime}(r)+p v_{0}^{-(p+1)}(r) v_{1}(r)-\frac{(N-2)}{3} r v_{0}^{\prime}(r)+\beta v_{0}(r)=0, \quad r \in(0, \infty), \\
v_{1}(0)=0, \quad v_{1}^{\prime}(0)=0 .
\end{array}\right.
$$

Then for $r \in\left[R_{0}, \infty\right)$,

$$
v_{1}(r)=C_{p} r^{2+\alpha}+r^{2-\frac{N-3}{2}}\left(a_{1} \cos (\omega \ln r)+b_{1} \sin (\omega \ln r)\right)+o\left(r^{2-\frac{N-3}{2}}\right),
$$

where $C_{p}$ satisfies

$$
\left[(2+\alpha)(N-1+\alpha)+p A_{p}^{-(p+1)}\right] C_{p}=A_{p}\left[\frac{2(N-2)}{3(p+1)}-\beta\right]
$$

$\left(a_{1}, b_{1}\right)$ is the solution of

$\left\{\begin{array}{l}D_{1} a_{1}+4 \omega b_{1}=-\beta a_{0}+\frac{(N-2)}{3} b_{0} \omega-\frac{(N-2)(N-3)}{6} a_{0}+p(p+1) A_{p}^{-(p+2)} C_{p} a_{0} \\ -4 \omega a_{1}+D_{1} b_{1}=-\beta b_{0}-\frac{(N-2)}{3} a_{0} \omega-\frac{(N-2)(N-3)}{6} b_{0}+p(p+1) A_{p}^{-(p+2)} C_{p} b_{0},\end{array}\right.$ where $D_{1}=\frac{(N+1)(7-N)}{4}-\omega^{2}+p A_{p}^{-(p+1)} ; a_{0}, b_{0}$ and $\omega$ are given in Lemma 2.1.

Proof. Let

$$
v_{1}(r)=C_{p} r^{2+\alpha}+h(r) r^{2-\frac{N-3}{2}}+o\left(r^{2-\frac{N-3}{2}}\right)
$$

where

$$
h(r)=c_{1} \cos (\omega \ln r)+c_{2} \sin (\omega \ln r) .
$$

Using the expression of $v_{0}(r)$ in $(2.4),(2.9)$ can be obtained by direct calculations. Note that

$$
O\left(r^{-\left(N-3+\frac{2}{p+1}\right)}\right)=o\left(r^{-\frac{(N-3)}{2}}\right) .
$$


Now we obtain the following proposition.

Proposition 2.3. Let $p>p_{c}(N-1)$ and $v(r)$ be a solution of (2.1). Then for $\epsilon>0$ sufficiently small,

$$
v(r)=v_{0}(r)+\sum_{k=1}^{\infty} \epsilon^{2 k} v_{k}(r) .
$$

Moreover, for $r \in\left[R_{0}, \infty\right)$,

$$
v_{k}(r)=\sum_{j=1}^{k} d_{j}^{k} r^{2 j+\alpha}+\sum_{j=1}^{k} e_{j}^{k} r^{2 j-\frac{N-3}{2}} \sin \left(\omega \ln r+E_{j}^{k}\right)+o\left(r^{2 k-\frac{N-3}{2}}\right),
$$

where $d_{j}^{k}, e_{j}^{k}, E_{j}^{k}(j=1,2, \ldots, k)$ are constants. Moreover,

$$
d_{1}^{1}=C_{p}, \quad e_{1}^{1}=\sqrt{a_{1}^{2}+b_{1}^{2}}, \quad \sin E_{1}^{1}=\frac{a_{1}}{e_{1}^{1}}, \quad \cos E_{1}^{1}=\frac{b_{1}}{e_{1}^{1}}
$$

where $C_{p}, a_{1}, b_{1}$ are given in Lemma 2.2.

Proof. Using the Taylor's expansion of $v^{-p}$ and the expressions of $v_{0}(r), v_{1}(r), \ldots, v_{k-1}(r)$, we can obtain this proposition by the induction argument and direct calculations. Note that

$$
O\left(r^{2-\frac{N-3}{2}}\right)=o\left(r^{2+\alpha}\right) .
$$

Now we obtain the following theorem.

Theorem 2.4. Let $p>p_{c}(N-1)$ and $w_{\epsilon}^{\text {inn }}(\theta)$ be an inner solution of $(1.10)$ with $w_{\epsilon}(0)=\epsilon^{\alpha}$. Then for any sufficiently small $\epsilon>0$ and $\theta>R_{0} \epsilon$ but $\theta$ is also sufficiently small,

$$
\begin{aligned}
w_{\epsilon}^{i n n}(\theta)=\quad & A_{p} \theta^{\alpha}+C_{p} \theta^{\alpha+2}+\sum_{k=2}^{\infty} \sum_{j=1}^{k} d_{j}^{k} \epsilon^{2(k-j)} \theta^{2 j+\alpha} \\
+ & \epsilon^{\frac{N-3}{2}+\alpha}\left[\frac{a_{0} \cos \left[\omega \ln \frac{\theta}{\epsilon}\right]+b_{0} \sin \left[\omega \ln \frac{\theta}{\epsilon}\right]}{\theta^{\frac{N-3}{2}}}+\frac{a_{1} \cos \left[\omega \ln \frac{\theta}{\epsilon}\right]+b_{1} \sin \left[\omega \ln \frac{\theta}{\epsilon}\right]}{\theta^{\frac{N-3}{2}-2}}\right. \\
& \left.+\sum_{k=2}^{\infty}\left(\sum_{j=1}^{k} e_{j}^{k} \epsilon^{2(k-j)} \theta^{2 j-\frac{N-3}{2}} \sin \left(\omega \ln \frac{\theta}{\epsilon}+E_{j}^{k}\right)+o\left(\theta^{2 k-\frac{N-3}{2}}\right)\right)\right] .
\end{aligned}
$$

Proof. This theorem can be obtained directly from Proposition 2.3 by setting $r=\theta / \epsilon$.

The following lemmas which are similar to Lemma 2.4 and Lemma 3.3 of [1] respectively will be useful in the subsequent proofs. 
Lemma 2.5. Let $p>p_{c}(N-1)$ and

$$
v(Q, \theta)=Q v_{0}\left(Q^{-\frac{p+1}{2}} \theta\right) .
$$

Then for $Q^{-\frac{p+1}{2}} \theta \geq R_{0}$, and for $n=0,1,2, v(Q, \theta)$ satisfies

(i) $\frac{\partial^{n}}{\partial Q^{n}}(v(Q, \theta))=\left\{\begin{array}{c}A_{p} \theta^{\alpha} \\ 0\end{array}\right\}$

$$
\begin{aligned}
& +\frac{\partial^{n}}{\partial Q^{n}}\left\{C \theta^{-\frac{N-3}{2}} Q^{\left(\frac{(p+1)(N-3)}{4}+1\right)} \sin \left(\omega \ln \left(Q^{-\frac{p+1}{2}} \theta\right)+D\right)\right\} \\
& +Q^{-n+\left[\frac{p+1}{2}(N-3+\alpha)+1\right]} O\left(\theta^{-(N-3+\alpha)}\right),
\end{aligned}
$$

(ii) $\frac{\partial^{n}}{\partial Q^{n}}\left(v_{\theta}^{\prime}(Q, \theta)\right)=\left\{\begin{array}{c}A_{p} \theta^{\alpha-1} \\ 0\end{array}\right\}$

$$
\begin{aligned}
& +\frac{\partial^{n+1}}{\partial Q^{n} \partial \theta}\left\{C \theta^{-\frac{N-3}{2}} Q^{\left(\frac{(p+1)(N-3)}{4}+1\right)} \sin \left(\omega \ln \left(Q^{-\frac{p+1}{2}} \theta\right)+D\right)\right\} \\
& +Q^{-n+\left[\frac{p+1}{2}(N-3+\alpha)+1\right]} O\left(\theta^{-(N-2+\alpha)}\right),
\end{aligned}
$$

where

$$
D=\tan ^{-1}\left(\frac{b_{0}}{a_{0}}\right), \quad C=\sqrt{a_{0}^{2}+b_{0}^{2}} .
$$

Proof. These estimates are obtained by the expansion of $v_{0}(r)$ given above and some calculations.

Lemma 2.6. In the region $\theta=\left|O\left(Q^{-\frac{\sigma}{(2-\sigma) \alpha}}\right)\right|$, the solution $w(Q, \theta)$ of (1.10) with $w(Q, 0)=Q, w_{\theta}^{\prime}(Q, 0)=0$ satisfies
(i) $\left|\frac{\partial w}{\partial Q}(Q, \theta)-\frac{\partial v}{\partial Q}(Q, \theta)\right|=Q^{\frac{(p+1)(N-3)}{4}}\left|o\left(\theta^{-\frac{N-3}{2}}\right)\right|$;
(ii) $\left|\frac{\partial w_{\theta}^{\prime}}{\partial Q}(Q, \theta)-\frac{\partial v_{\theta}^{\prime}}{\partial Q}(Q, \theta)\right|=Q^{\frac{(p+1)(N-3)}{4}}\left|o\left(\theta^{-\frac{N-1}{2}}\right)\right|$;
(iii) $\left|\frac{\partial^{2} w}{\partial Q^{2}}(Q, \theta)-\frac{\partial^{2} v}{\partial Q^{2}}(Q, \theta)\right|=Q^{\left(\frac{(p+1)(N-3)}{4}-1\right)}\left|o\left(\theta^{-\frac{N-3}{2}}\right)\right|$;
(iv) $\left|\frac{\partial^{2} w_{\theta}^{\prime}}{\partial Q^{2}}(Q, \theta)-\frac{\partial^{2} v_{\theta}^{\prime}}{\partial Q^{2}}(Q, \theta)\right|=Q^{\left(\frac{(p+1)(N-3)}{4}-1\right)}\left|o\left(\theta^{-\frac{N-1}{2}}\right)\right|$.

Proof. This lemma can be obtained from Lemma 2.5 and Theorem 2.4. Note that

Moreover,

$$
\epsilon=Q^{\frac{1}{\alpha}}, \quad \frac{\sigma}{\alpha}=-\frac{(p+1)(N-3)}{4}-1
$$

provided $Q$ suitably small.

$$
Q^{-\frac{p+1}{2}} \theta=\left|O\left(Q^{-\frac{p+1}{2-\sigma}}\right)\right|>R_{0}
$$

Now we can write the inner solution obtained in Theorem 2.4 in the form of parameter $Q$ : 
Theorem 2.7. Let $p>p_{c}(N-1)$ and $w_{Q}^{i n n}(\theta)$ be an inner solution of $(1.10)$ with $w_{Q}(0)=Q$. Then for any sufficiently large $Q>0$ and $\theta=\left|O\left(Q^{-\frac{\sigma}{(2-\sigma) \alpha}}\right)\right|$,

$$
\begin{aligned}
& w_{Q}^{i n n}(\theta) \\
& =A_{p} \theta^{\alpha}+C_{p} \theta^{\alpha+2}+\sum_{k=2}^{\infty} \sum_{j=1}^{k} d_{j}^{k} Q^{(p+1)(k-j)} \theta^{2 j+\alpha} \\
& +Q^{-\frac{\sigma}{\alpha}}\left[\frac{a_{0} \cos \left[\omega \ln \left(Q^{-\frac{p+1}{2}} \theta\right)\right]+b_{0} \sin \left[\omega \ln \left(Q^{-\frac{p+1}{2}} \theta\right)\right]}{\theta^{\frac{N-3}{2}}}\right. \\
& \quad+\frac{a_{1} \cos \left[\omega \ln \left(Q^{-\frac{p+1}{2}} \theta\right)\right]+b_{1} \sin \left[\omega \ln \left(Q^{-\frac{p+1}{2}} \theta\right)\right]}{\theta^{\frac{N-3}{2}-2}} \\
& \left.\quad+\sum_{k=2}^{\infty}\left(\sum_{j=1}^{k} e_{j}^{k} Q^{(p+1)(k-j)} \theta^{2 j-\frac{N-3}{2}} \sin \left(\omega \ln \left(Q^{-\frac{p+1}{2}} \theta\right)+E_{j}^{k}\right)+o\left(\theta^{2 k-\frac{N-3}{2}}\right)\right)\right] .
\end{aligned}
$$

\section{OUTER SOLUTIONS}

In this section we study the asymptotic behaviors of solutions $w(\theta)$ of (1.10) far from $\theta=0$.

Let $w_{*}(\theta)$ be the singular solution given in (1.11). We first obtain the following lemma.

\section{Lemma 3.1. Equation}

$$
\frac{1}{\sin ^{N-2} \theta} \frac{d}{d \theta}\left(\sin ^{N-2} \theta \frac{d \phi}{d \theta}(\theta)\right)+\beta \phi(\theta)+p w_{*}^{-(p+1)}(\theta) \phi(\theta)=0, \quad 0<\theta<\frac{\pi}{2},
$$

admits two fundamental solutions $\phi_{1}(\theta)$ and $\phi_{2}(\theta)$. Moreover, any solution $\phi(\theta)$ of (3.1) can be written in the form

$$
\phi(\theta)=c_{1} \phi_{1}(\theta)+c_{2} \phi_{2}(\theta), \text { where } c_{1} \text { and } c_{2} \text { are constants, }
$$

which satisfies that as $\theta \rightarrow 0$,

$$
\phi(\theta)=\theta^{-\frac{N-3}{2}}\left[c_{1} \cos \left(\omega \ln \frac{\theta}{2}\right)+c_{2} \sin \left(\omega \ln \frac{\theta}{2}\right)\right]+O\left(\theta^{2-\frac{N-3}{2}}\right) .
$$

Proof. Let $\tilde{\phi}(\theta)=[\sin \theta]^{-\alpha} \phi(\theta)$. We see that $\tilde{\phi}(\theta)$ satisfies the equation

(3.3) $\sin ^{2} \theta \tilde{\phi}^{\prime \prime}(\theta)+(N-2+2 \alpha) \sin \theta \cos \theta \tilde{\phi}^{\prime}(\theta)+(p+1) A_{p}^{-(p+1)} \tilde{\phi}(\theta)=0$.

Under the Emden-Fowler transformations:

$$
\psi(t)=\tilde{\phi}(\theta), \quad t=\ln \tan \frac{\theta}{2},
$$


we obtain that for $t \in(-\infty, 0)$,

$$
\psi^{\prime \prime}(t)+(N-3+2 \alpha)\left(1-\frac{2 e^{2 t}}{1+e^{2 t}}\right) \psi^{\prime}(t)+2(N-3+\alpha) \psi(t)=0 .
$$

Note that

$$
\sin \theta=\frac{2 e^{t}}{1+e^{2 t}}, \quad \cos \theta=\frac{1-e^{2 t}}{1+e^{2 t}}=1-\frac{2 e^{2 t}}{1+e^{2 t}} .
$$

We can obtain solutions of (3.4) by shooting backwards under the conditions $\psi(0)=a, \psi^{\prime}(0)=0$. The standard ODE arguments imply that (3.4) admits two fundamental solutions $\psi_{1}, \psi_{2} \in C^{2}(-\infty, 0)$ such that any solution $\psi(t)$ of (3.4) satisfies

$$
\psi(t)=\ell_{1} \psi_{1}(t)+\ell_{2} \psi_{2}(t)
$$

where $\ell_{1}$ and $\ell_{2}$ are two constants. Now we show that as $t \rightarrow-\infty$,

$$
\psi(t)=e^{\sigma t}\left[\ell_{3} \cos \omega t+\ell_{4} \sin \omega t\right]+O\left(e^{(\sigma+2) t}\right)
$$

where $\sigma=-\left(\frac{N-3}{2}+\alpha\right)$.

We see that the characteristic equation of (3.4) admits a pair roots $\lambda_{1}=\sigma+i \omega, \lambda_{2}=\sigma-i \omega$ as $t \rightarrow-\infty$ since

$$
(N-3+2 \alpha)^{2}-8(N-3+\alpha)<0 \text { for } p>p_{c}(N-1) .
$$

By the standard argument of variation of constants we obtain the following integral equation

$$
\psi(t)=e^{\sigma t}\left[\ell_{3} \cos \omega t+\ell_{4} \sin \omega t\right]+\frac{1}{\omega} \int_{T}^{t} e^{\sigma\left(t-t^{\prime}\right)} \sin \omega\left(t-t^{\prime}\right) j(\psi)\left(t^{\prime}\right) d t^{\prime},
$$

where $T \in(-\infty, 0)$ with sufficiently large $|T|, j(\psi)\left(t^{\prime}\right)=-(N-3+$ $2 \alpha) \frac{2 e^{2 t^{\prime}}}{1+e^{2 t^{\prime}}} \psi^{\prime}\left(t^{\prime}\right)$. Setting $\hat{\psi}(t)=e^{-\sigma t} \psi(t)$, we see

$$
\hat{\psi}(t)=\left[\ell_{3} \cos \omega t+\ell_{4} \sin \omega t\right]+\frac{1}{\omega} \int_{T}^{t} \sin \omega\left(t-t^{\prime}\right) j(\hat{\psi})\left(t^{\prime}\right) d t^{\prime},
$$

where

$$
j(\hat{\psi})\left(t^{\prime}\right)=-(N-3+2 \alpha) \frac{2 e^{2 t^{\prime}}}{1+e^{2 t^{\prime}}}\left(\sigma \hat{\psi}\left(t^{\prime}\right)+\hat{\psi}^{\prime}\left(t^{\prime}\right)\right) .
$$

It follows from (3.5) that

$$
\left\|\hat{\psi}-\left[\ell_{3} \cos \omega t+\ell_{4} \sin \omega t\right]\right\|_{0} \leq \tau\left(|\sigma|\|\hat{\psi}\|_{0}+\left\|\hat{\psi}^{\prime}\right\|_{0}\right),
$$

where $0<\tau:=\tau(T) \rightarrow 0$ as $T \rightarrow-\infty$ and $\|\rho\|_{0}=\sup _{-\infty<t<T}|\rho(t)|$.

On the other hand, we see that $z(t):=\psi^{\prime}(t)$ satisfies the equation

$$
z^{\prime \prime}(t)+(N-3+2 \alpha) z^{\prime}(t)+2(N-3+\alpha) z(t)+h\left(t, \psi(t), \psi^{\prime}(t)\right)=0
$$


where

$$
\begin{aligned}
& h\left(t, \psi(t), \psi^{\prime}(t)\right)=(N-3+2 \alpha)^{2} \frac{2 e^{2 t}}{\left(1+e^{2 t}\right)}\left(1-\frac{2 e^{2 t}}{\left(1+e^{2 t}\right)}\right) \psi^{\prime}(t) \\
& \quad-2(N-3+2 \alpha) \frac{2 e^{2 t}}{\left(1+e^{2 t}\right)^{2}} \psi^{\prime}(t)+2(N-3+\alpha)(N-3+2 \alpha) \frac{2 e^{2 t}}{\left(1+e^{2 t}\right)} \psi(t) .
\end{aligned}
$$

Thus,

$e^{-\sigma t} \psi^{\prime}(t)=\left[\ell_{5} \cos \omega t+\ell_{6} \sin \omega t\right]+\frac{1}{\omega} \int_{T}^{t} \sin \omega\left(t-t^{\prime}\right) h\left(t^{\prime}, \hat{\psi}\left(t^{\prime}\right), \hat{\psi}^{\prime}\left(t^{\prime}\right)\right) d t^{\prime}$

where

$$
\ell_{5}=\ell_{3} \sigma+\ell_{4} \omega, \quad \ell_{6}=\ell_{4} \sigma-\omega \ell_{3}
$$

and

$$
\begin{aligned}
& h\left(t, \hat{\psi}(t), \hat{\psi}^{\prime}(t)\right)=(N-3+2 \alpha)^{2} \frac{2 e^{2 t}}{\left(1+e^{2 t}\right)}\left(1-\frac{2 e^{2 t}}{\left(1+e^{2 t}\right)}\right)\left(\sigma \hat{\psi}(t)+\hat{\psi}^{\prime}(t)\right) \\
& -2(N-3+2 \alpha) \frac{2 e^{2 t}}{\left(1+e^{2 t}\right)^{2}}\left(\sigma \hat{\psi}(t)+\hat{\psi}^{\prime}(t)\right) \\
& +2(N-3+\alpha)(N-3+2 \alpha) \frac{2 e^{2 t}}{\left(1+e^{2 t}\right)} \hat{\psi}(t) .
\end{aligned}
$$

It follows from (3.7) that

$$
\left\|e^{-\sigma t} \psi^{\prime}(t)-\left[\ell_{5} \cos \omega t+\ell_{6} \sin \omega t\right]\right\|_{0} \leq \tau\left(|\sigma|\|\hat{\psi}\|_{0}+\left\|\hat{\psi}^{\prime}\right\|_{0}\right),
$$

where $\tau$ is as in (3.6). Since $\hat{\psi}^{\prime}(t)=e^{-\sigma t} \psi^{\prime}(t)-\sigma \hat{\psi}(t)$, it follows from (3.6) and (3.8) that by choosing $|T|$ suitably large,

$$
\|\hat{\psi}\|_{0} \leq C, \quad\left\|\hat{\psi}^{\prime}\right\|_{0} \leq C
$$

where $C=C\left(p, N, T, \ell_{3}, \ell_{4}\right)$. Both (3.9) and (3.5) imply that as $t \rightarrow$ $-\infty$,

$$
\hat{\psi}(t)=\left[\ell_{3} \cos \omega t+\ell_{4} \sin \omega t\right]+O\left(e^{2 t}\right) .
$$

Therefore, as $t \rightarrow-\infty$,

$$
\psi(t)=e^{\sigma t}\left[\ell_{3} \cos \omega t+\ell_{4} \sin \omega t\right]+O\left(e^{(\sigma+2) t}\right) .
$$

This implies that as $\theta \rightarrow 0^{+}$,

$\phi(\theta)=[\sin \theta]^{\alpha}\left(\tan \frac{\theta}{2}\right)^{\sigma}\left[\ell_{3} \cos \left(\omega \ln \frac{\theta}{2}\right)+\ell_{4} \sin \left(\omega \ln \frac{\theta}{2}\right)+O\left(\theta^{2}\right)\right]+O\left([\sin \theta]^{\alpha}\left(\tan \frac{\theta}{2}\right)^{\sigma+2}\right)$.

Note that for sufficiently small $x>0, \tan x=x+O\left(x^{3}\right)$ and $\ln (\tan x)=$ $\ln \left(x+O\left(x^{3}\right)\right)=\ln x+O\left(x^{2}\right)$. The Taylor's expansions of $\sin \theta$ and $\tan \frac{\theta}{2}$ imply that (3.2) holds. This completes the proof. 
Remark 3.2. For any $\delta>0$ sufficiently small, if $c_{1}$ and $c_{2}$ in (3.2) satisfy that $c_{1}=\tilde{c}_{1} \delta, c_{2}=\tilde{c}_{2} \delta$, where $\tilde{c}_{1}$ and $\tilde{c}_{2}$ are constants, then as $\theta \rightarrow 0^{+}$,

$$
\phi(\theta):=\phi_{\delta}(\theta)=\delta \theta^{-\frac{N-3}{2}}\left[\tilde{c}_{1} \cos \left(\omega \ln \frac{\theta}{2}\right)+\tilde{c}_{2} \sin \left(\omega \ln \frac{\theta}{2}\right)\right]+O(\delta) \theta^{2-\frac{N-3}{2}} .
$$

Indeed, if $\ell_{3}=\tilde{\ell}_{3} \delta, \ell_{4}=\tilde{\ell}_{4} \delta$, where $\tilde{\ell}_{3}$ and $\tilde{\ell}_{4}$ are constants, we see from (3.8) that

$$
|\sigma|\|\hat{\psi}\|_{0}+\left\|\hat{\psi}^{\prime}\right\|_{0} \leq C \delta
$$

where $C:=C\left(p, N, T, \tilde{\ell}_{3}, \tilde{\ell}_{4}\right)>0$ is independent of $\delta$. Hence

$$
\psi(t):=\psi_{\delta}(t)=e^{\sigma t} \delta\left[\tilde{\ell}_{3} \cos \omega t+\tilde{\ell}_{4} \sin \omega t\right]+O(\delta) e^{(\sigma+2) t} .
$$

For any $\delta>0$ sufficiently small, if $w \in C^{2}\left(0, \frac{\pi}{2}\right)$ is a solution of (1.10) and

$$
w(\theta)=w_{*}(\theta)+\delta \phi_{\delta}(\theta)+\delta^{2} \psi_{\delta}(\theta)
$$

where

$$
\phi_{\delta}(\theta)=\tilde{c}_{1} \delta \phi_{1}(\theta)+\tilde{c}_{2} \delta \phi_{2}(\theta)
$$

is a solution of (3.1) with

$$
c_{1}=\tilde{c}_{1} \delta, \quad c_{2}=\tilde{c}_{2} \delta,
$$

then $\psi_{\delta}(\theta)$ satisfies the problem

(3.14)

$$
\left\{\begin{array}{l}
\frac{1}{\sin ^{N-2} \theta} \frac{d}{d \theta}\left(\sin ^{N-2} \theta \frac{d \psi}{d \theta}(\theta)\right)+\beta \psi(\theta)+p w_{*}^{-(p+1)} \psi(\theta) \\
\quad+\delta^{-2}\left[\left(w_{*}+\delta \phi_{\delta}+\delta^{2} \psi\right)^{-p}-w_{*}^{-p}+p w_{*}^{-(p+1)} \delta \phi_{\delta}+\delta^{2} p w_{*}^{-(p+1)} \psi\right]=0, \quad 0<\theta<\pi / 2, \\
\psi^{\prime}\left(\frac{\pi}{2}\right)=-\left(\tilde{c}_{1} \phi_{1}^{\prime}\left(\frac{\pi}{2}\right)+\tilde{c}_{2} \phi_{2}^{\prime}\left(\frac{\pi}{2}\right)\right) .
\end{array}\right.
$$

Lemma 3.3. For any $\delta>0$ sufficiently small and each fixed pair $\left(\tilde{c}_{1}, \tilde{c}_{2}\right)$, (3.14) admits solutions $\psi_{\delta} \in C^{2}(0, \pi / 2)$.

Proof. We set the initial value conditions on $\psi$ of (3.14) at $\theta=\pi / 2$ : $\psi(\pi / 2)=1$ provided

$$
\psi^{\prime}\left(\frac{\pi}{2}\right)=-\left(\tilde{c}_{1} \phi_{1}^{\prime}\left(\frac{\pi}{2}\right)+\tilde{c}_{2} \phi_{2}^{\prime}\left(\frac{\pi}{2}\right)\right)=0
$$

$\psi(\pi / 2)=0$ provided

$$
\psi^{\prime}\left(\frac{\pi}{2}\right)=-\left(\tilde{c}_{1} \phi_{1}^{\prime}\left(\frac{\pi}{2}\right)+\tilde{c}_{2} \phi_{2}^{\prime}\left(\frac{\pi}{2}\right)\right) \neq 0
$$

Then, the standard shooting argument in ODE implies that (3.14) admits a unique nontrivial solution $\psi_{\delta}$ in $C^{2}(0, \pi / 2)$. Note that there is no singularity of $(3.14)$ for $\theta \in(0, \pi / 2)$. Note also that $\psi_{\delta}$ depends on $\tilde{c}_{1}$ and $\tilde{c}_{2}$.

Now we obtain the following proposition. 
Proposition 3.4. For any $\delta>0$ sufficiently small and $\psi_{\delta}$ being given in Lemma 3.3, then for $\theta=\left|O\left(\delta^{\frac{2}{(2-\sigma)}}\right)\right|$,

$$
\psi_{\delta}(\theta)=\theta^{-\frac{N-3}{2}}\left[\tilde{d}_{1} \cos \left[\omega \ln \frac{\theta}{2}\right]+\tilde{d}_{2} \sin \left[\omega \ln \frac{\theta}{2}\right]\right]+O\left(\theta^{2-\frac{N-3}{2}}\right)
$$

where $\tilde{d}_{1}$ and $\tilde{d}_{2}$ are constants depending on $\tilde{c}_{1}$ and $\tilde{c}_{2}$ but independent of $\delta$.

Proof. Setting $\psi_{\delta}(\theta)=[\sin \theta]^{\alpha} \tilde{\psi}_{\delta}(\theta)$, we see that $\tilde{\psi}_{\delta}(\theta)$ satisfies the problem

$\left\{\begin{array}{l}\sin ^{2} \theta \tilde{\psi}^{\prime \prime}(\theta)+(N-3+2 \alpha) \cos \theta \sin \theta \tilde{\psi}^{\prime}(\theta)+2(N-3+\alpha) \tilde{\psi}(\theta)+G(\tilde{\psi}(\theta))=0, \\ \tilde{\psi}^{\prime}\left(\frac{\pi}{2}\right)=\psi_{\delta}^{\prime}\left(\frac{\pi}{2}\right),\end{array}\right.$

where

$$
\begin{aligned}
G(\tilde{\psi}(\theta)) & =[\sin \theta]^{2-\alpha} \delta^{-2}\left[w_{*}(\theta)+\delta \phi_{\delta}(\theta)+\delta^{2}[\sin \theta]^{\alpha} \tilde{\psi}(\theta)\right]^{-p} \\
& -w_{*}^{-p}+p w_{*}^{-(p+1)} \delta \phi_{\delta}(\theta)+\delta^{2} p w_{*}^{-(p+1)}[\sin \theta]^{\alpha} \tilde{\psi}(\theta) .
\end{aligned}
$$

Under the Emden-Fowler transformations:

$$
z(t)=\tilde{\psi}(\theta), \quad t=\ln \tan \frac{\theta}{2}
$$

we obtain

$$
z^{\prime \prime}(t)+(N-3+2 \alpha)\left(1-\frac{2 e^{2 t}}{1+e^{2 t}}\right) z^{\prime}(t)+2(N-3+\alpha) z(t)+G(z(t))=0 .
$$

By the standard argument of variation of constants and Lemma 3.1, if

$$
\tilde{\phi}_{1}(t)=[\sin \theta]^{-\alpha} \phi_{1}(\theta), \quad \tilde{\phi}_{2}(t)=[\sin \theta]^{-\alpha} \phi_{2}(\theta),
$$


then we obtain the following integral equation for $T \in(-\infty, 0)$ and $|T|$ suitably large,

$$
\begin{aligned}
z(t)=\vartheta_{1} \tilde{\phi}_{1}(t) & +\vartheta_{2} \tilde{\phi}_{2}(t)+\int_{T}^{t} \frac{-\tilde{\phi}_{1}(t) \tilde{\phi}_{2}\left(t^{\prime}\right)+\tilde{\phi}_{2}(t) \tilde{\phi}_{1}\left(t^{\prime}\right)}{\tilde{\phi}_{1}\left(t^{\prime}\right) \tilde{\phi}_{2}^{\prime}\left(t^{\prime}\right)-\tilde{\phi}_{1}^{\prime}\left(t^{\prime}\right) \tilde{\phi}_{2}\left(t^{\prime}\right)} d t^{\prime} \\
=e^{\sigma t}\left[\vartheta_{1}\right. & \left.\cos \omega t+\vartheta_{2} \sin \omega t\right]+O\left(e^{(\sigma+2) t}\right) \\
& +\frac{1}{\omega} \int_{T}^{t} e^{\sigma\left(t-t^{\prime}\right)} \frac{\sin \omega\left(t-t^{\prime}\right)+O\left(e^{2 t^{\prime}}\right)}{1+O\left(e^{2 t^{\prime}}\right)} G\left(z\left(t^{\prime}\right)\right) d t^{\prime} \\
=e^{\sigma t}\left[\vartheta_{1}\right. & \left.\cos \omega t+\vartheta_{2} \sin \omega t\right]+O\left(e^{(\sigma+2) t}\right) \\
& +\frac{p(p+1)}{2 \omega} \int_{T}^{t} e^{\sigma t} \sin \omega\left(t-t^{\prime}\right)\left[e^{\sigma t^{\prime}} \delta^{2}\right]\left[\rho\left(t^{\prime}\right)\right]^{2} d t^{\prime} \\
& +\frac{1}{\omega} \int_{T}^{t} e^{\sigma t} \sin \omega\left(t-t^{\prime}\right) O\left(\left[e^{\sigma t^{\prime}} \delta^{2}\right]^{2}\left[\rho\left(t^{\prime}\right)\right]^{3}\right) d t^{\prime} \\
& +\frac{1}{\omega} \int_{T}^{t} e^{\sigma t} \sin \omega\left(t-t^{\prime}\right) O\left(e^{2 t^{\prime}}\right)\left[e^{\sigma t^{\prime}} \delta^{2}\right]\left[\rho\left(t^{\prime}\right)\right]^{2} d t^{\prime} \\
& +\frac{1}{\omega} \int_{T}^{t} e^{\sigma t} \sin \omega\left(t-t^{\prime}\right) O\left(e^{2 t^{\prime}}\right) O\left(\left[e^{\sigma t^{\prime}} \delta^{2}\right]^{2}\left[\rho\left(t^{\prime}\right)\right]^{3}\right) d t^{\prime}
\end{aligned}
$$

where

$$
\rho\left(t^{\prime}\right)=\left(\tilde{c}_{1} \cos \omega t^{\prime}+\tilde{c}_{2} \sin \omega t^{\prime}\right)+e^{-\sigma t^{\prime}} z\left(t^{\prime}\right) .
$$

Setting $\hat{z}(t)=e^{-\sigma t} z(t)$, arguments similar to those in the proof of Lemma 3.1 imply that there exists $C:=C(N, p, T)>0$ but independent of $\delta$ such that

$$
\left\|\hat{z}-\left[\vartheta_{1} \cos \omega t+\vartheta_{2} \sin \omega t\right]\right\|_{0} \leq C
$$

provided that for $t \in[2 T, 10 T]$,

$$
\delta^{2}=\left|O\left(e^{(2-\sigma) t}\right)\right| .
$$

Therefore,

$$
z(t)=e^{\sigma t}\left[\vartheta_{1} \cos \omega t+\vartheta_{2} \sin \omega t\right]+O\left(e^{(\sigma+2) t}\right)
$$

provided that (3.19) holds. Therefore,

$\psi_{\delta}(\theta)=[\sin \theta]^{\alpha}\left(\tan \frac{\theta}{2}\right)^{\sigma}\left[\vartheta_{1} \cos \left[\omega \ln \frac{\theta}{2}\right]+\vartheta_{2} \sin \left[\omega \ln \frac{\theta}{2}\right]+O\left(\theta^{2}\right)\right]+O\left([\sin \theta]^{\alpha}\left(\tan \frac{\theta}{2}\right)^{\sigma+2}\right)$

provided

$$
\theta=\left|O\left(\delta^{\frac{2}{2-\sigma}}\right)\right|
$$

The Taylor's expansions of $\sin \theta$ and $\tan \frac{\theta}{2}$ imply that (3.15) holds provided that (3.22) holds. This completes the proof of this proposition. 
Now we are in the position to obtain the following theorem.

Theorem 3.5. For any $\delta>0$ sufficiently small, problem (1.10) admits outer solutions $w_{\delta}^{\text {out }} \in C^{2}(0, \pi / 2)$ satisfying

$$
w_{\delta}^{\text {out }}(\theta)=w_{*}(\theta)+\delta \phi_{\delta}(\theta)+\delta^{2} \psi_{\delta}(\theta), \quad \theta \in\left(0, \frac{\pi}{2}\right), \quad w_{\delta}^{\prime}\left(\frac{\pi}{2}\right)=0 .
$$

\section{Moreover,}

$$
w_{\delta}^{\text {out }}(\theta)=A_{p} \theta^{\alpha}-\frac{A_{p}}{3(p+1)} \theta^{\alpha+2}+\delta^{2}\left[\frac{\vartheta_{3} \cos \left(\omega \ln \frac{\theta}{2}\right)+\vartheta_{4} \sin \left(\omega \ln \frac{\theta}{2}\right)}{\theta^{\frac{N-3}{2}}}+O\left(\frac{1}{\theta^{\frac{N-3}{2}-2}}\right)\right]
$$

provided

$$
\theta=\left|O\left(\delta^{\frac{2}{2-\sigma}}\right)\right|,
$$

where $\vartheta_{3}$ and $\vartheta_{4}$ are constants which are independent of $\delta$.

Proof. This theorem can be obtained from the expression of $w(\theta)$, (3.21) and the Taylor's expansions of $\sin \theta$ and $\tan \frac{\theta}{2}$. Note that for $\delta>0$ sufficiently small and $\theta=\left|O\left(\delta^{\frac{2}{2-\sigma}}\right)\right|$,

$$
O\left(\theta^{4+\alpha}\right)=o\left(\delta^{2} \theta^{2-\frac{N-3}{2}}\right) .
$$

Remark 3.6. It is know from (3.19) that $\delta^{2}=\left|O\left(\theta^{2-\sigma}\right)\right|$. Thus $w_{\delta}^{\text {out }}$ can also be expressed by

$$
\begin{gathered}
w_{\delta}^{\text {out }}(\theta)=A_{p} \theta^{\alpha}-\frac{A_{p}}{3(p+1)} \theta^{\alpha+2}+\delta^{2}\left[\frac{\vartheta_{3} \cos \left[\omega \ln \frac{\theta}{2}\right]+\vartheta_{4} \sin \left[\omega \ln \frac{\theta}{2}\right]}{\theta^{\frac{N-3}{2}}}+\delta^{2} O\left(\theta^{\sigma-\frac{N-3}{2}}\right)\right] . \\
\text { 4. Infinitely MANy SOlutions of (1.10) AND PRoof OF } \\
\text { TheOREM 1.1 }
\end{gathered}
$$

In this section we will construct infinitely many regular solutions for (1.10) by combining the inner and outer solutions.

We first construct a solution of problem

$$
\left\{\begin{array}{l}
\frac{1}{\sin ^{N-2} \theta} \frac{d}{d \theta}\left(\sin ^{N-2} \theta \frac{d w}{d \theta}(\theta)\right)+\beta w(\theta)-w^{-p}(\theta)=0, \quad w(\theta)>0, \quad 0<\theta<\frac{\pi}{2}, \\
w(0)=Q\left(:=\epsilon^{\alpha}\right), \quad w_{\theta}^{\prime}\left(\frac{\pi}{2}\right)=0
\end{array}\right.
$$

by using the expressions in Theorems 2.7 and 3.5. The variables $Q$ and $\delta$ are then chosen to ensure that, at a fixed $\theta=\Theta$ chosen to satisfy

$$
\Theta=O\left(Q^{-\frac{\sigma}{(2-\sigma) \alpha}}\right)
$$

such that

$$
w_{Q}^{\text {inn }}(\Theta)=w_{\delta}^{\text {out }}(\Theta)
$$


and

$$
\left.\left[w_{Q}^{\text {inn }}(\theta)-w_{\delta}^{\text {out }}(\theta)\right]_{\theta}^{\prime}\right|_{\theta=\Theta}=0
$$

These can be done by arguments similar to those in the proof of Lemma 6.1 of [1]. From the choice of $Q$ and $\delta$ we deduce the existence of a $C^{2}$ function $w(\theta)$ defined by $w(\theta)=w_{Q}^{\operatorname{inn}}(\theta)$ for $\theta \leq \Theta$ and by $w(\theta)=$ $w_{\delta}^{\text {out }}(\theta)$ for $\theta \geq \Theta$. Thus $w(\theta)$ satisfies (4.1).

We first see that

$$
-\frac{A_{p}}{3(p+1)}=C_{p}
$$

Note that

$$
\begin{aligned}
(2+\alpha) & (N-1+\alpha)+p A_{p}^{-(p+1)} \\
= & \frac{2(p+2)}{p+1}\left(\frac{2(p+2)}{p+1}+N-3\right)+\frac{2 p}{p+1}\left(N-3+\frac{2}{p+1}\right) \\
= & \frac{4\left(p^{2}+5 p+4\right)}{(p+1)^{2}}+4(N-3) \\
= & 4\left(N-2+\frac{3}{p+1}\right) . \\
& \frac{2(N-2)}{3(p+1)}-\beta=-\frac{4}{3(p+1)}\left(N-2+\frac{3}{p+1}\right) .
\end{aligned}
$$

It follows from (2.10) that (4.2) holds.

Define $Q_{*}$ and $\delta_{*}^{2}$ by

$$
\omega \ln Q_{*}^{-\frac{(p+1)}{2}}+D=\omega \ln 2^{-1}+\phi+2 m \pi,
$$

with $m>1$ being an integer suitably large,

$$
\delta_{*}^{2}=\sqrt{\frac{a_{0}^{2}+b_{0}^{2}}{\vartheta_{3}^{2}+\vartheta_{4}^{2}}} Q_{*}^{-\frac{\sigma}{\alpha}},
$$

where $\phi$ satisfies that

Note that

$$
\phi=\tan ^{-1}\left(\frac{\vartheta_{4}}{\vartheta_{3}}\right)
$$

$$
O\left(\delta_{*}^{\frac{2}{2-\sigma}}\right)=O\left(Q_{*}^{-\frac{\sigma}{\alpha(2-\sigma)}}\right)
$$

$a_{0} \cos \left[\omega \ln \left(Q^{-\frac{(p+1)}{2}} \theta\right)\right]+b_{0} \sin \left[\omega \ln \left(Q^{-\frac{(p+1)}{2}} \theta\right)\right]=\sqrt{a_{0}^{2}+b_{0}^{2}} \sin \left(\omega \ln \theta+\omega \ln Q^{-\frac{(p+1)}{2}}+D\right)$,

$$
\vartheta_{3} \cos \left[\omega \ln \frac{\theta}{2}\right]+\vartheta_{4} \sin \left[\omega \ln \frac{\theta}{2}\right]=\sqrt{\vartheta_{3}^{2}+\vartheta_{4}^{2}} \sin \left(\omega \ln \theta+\omega \ln 2^{-1}+\phi\right) .
$$


Then we claim that the values of $Q$ and $\delta^{2}$ required to satisfy these conditions may be obtained as small perturbations of the values of $Q_{*}$ and $\delta_{*}^{2}$ given in (4.3) and (4.4), i.e.,

$$
\begin{aligned}
& Q=Q_{*}\left(1+O\left(Q_{*}^{-\frac{2 \sigma}{(2-\sigma) \alpha}}\right)\right), \\
& \delta^{2}=\delta_{*}^{2}\left(1+O\left(Q_{*}^{-\frac{2 \sigma}{(2-\sigma) \alpha}}\right)\right) .
\end{aligned}
$$

To show this we define the function $\mathbf{F}\left(Q, \delta^{2}\right)$ by

$$
\mathbf{F}^{T}\left(Q, \delta^{2}\right)=\left(\Theta^{\frac{N-3}{2}}\left(w_{Q}^{\text {inn }}(\Theta)-w_{\delta}^{\text {out }}(\Theta)\right),\left.\quad\left[\theta^{\frac{N-3}{2}}\left(w_{Q}^{\text {inn }}(\theta)-w_{\delta}^{\text {out }}(\theta)\right)\right]_{\theta}^{\prime}\right|_{\theta=\Theta}\right) \text {. }
$$

(We treat $\delta^{2}$ as a new variable.) Taking $Q=Q_{*}$ and $\delta^{2}=\delta_{*}^{2}$ we find a bound for $\mathbf{F}\left(Q_{*}, \delta_{*}^{2}\right)$ by making use of the behavior of $w_{Q}^{\text {inn }}(\theta)$ determined by Theorem 2.7, and the behavior of $w_{\delta}^{\text {out }}(\theta)$ given in Theorem 3.5. Accordingly we find for some $M>1$ suitably large,

$$
\left|\Theta^{-\frac{N-3}{2}} \mathbf{F}\left(Q_{*}, \delta_{*}^{2}\right)\right| \leq M \delta_{*}^{4} \Theta^{\sigma-\frac{N-3}{2}}+\text { small terms. }
$$

We now seek values of $Q$ and $\delta^{2}$ which are small perturbations of $Q_{*}$ and $\delta_{*}^{2}$ and for which $\mathbf{F}\left(Q, \delta^{2}\right)=0$. As in [1], we need to evaluate the Jacobian of $\mathbf{F}$ at $\left(Q_{*}, \delta_{*}^{2}\right)$. We can obtain the following estimates from Lemmas 2.5, 2.6 and Theorems 2.7, 3.5:

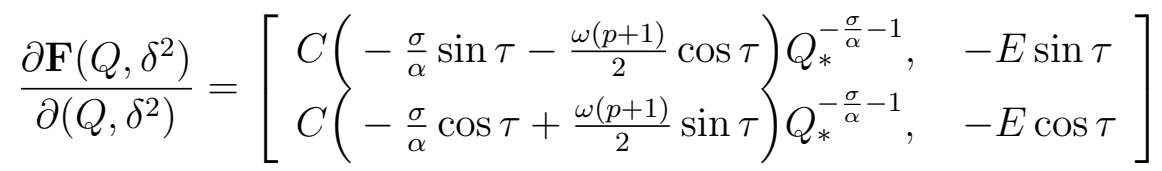

$$
\begin{aligned}
& \text { +small order terms, }
\end{aligned}
$$

where

$$
\begin{gathered}
C=\sqrt{a_{0}^{2}+b_{0}^{2}}, \quad E=\sqrt{\vartheta_{3}^{2}+\vartheta_{4}^{2}}, \\
\tau=\omega \ln \Theta+\omega \ln Q_{*}^{-\frac{(p+1)}{2}}+D=\omega \ln \Theta+\omega \ln 2^{-1}+\phi+2 m \pi .
\end{gathered}
$$

Note that

$$
-\frac{\sigma}{\alpha}-1=\frac{(N-3)(p+1)}{4} .
$$

To simplify this expression we define the function $\mathbf{G}(x, y)$ by

$$
\mathbf{G}(x, y)=\mathbf{F}\left(Q_{*}+x Q_{*}^{1+\frac{\sigma}{\alpha}}, \delta_{*}^{2}+y\right) .
$$


Using the bounds for $\mathbf{F}$ given in (4.9) and (3.26) and the results in Lemmas 2.5, 2.6, we express $\mathbf{G}(x, y)$ in the form

$$
\begin{aligned}
& \mathbf{G}(x, y)
\end{aligned}
$$

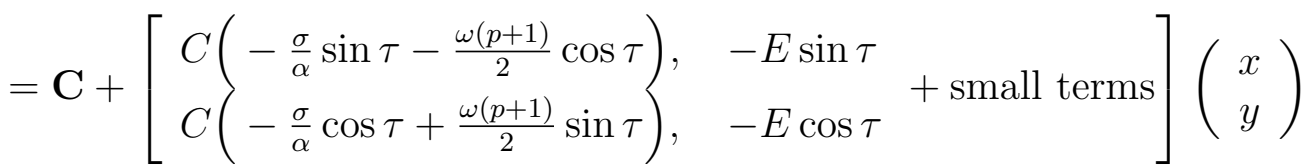

$$
\begin{aligned}
& +\mathbf{E}\left(x^{2}\left(\delta_{*}^{2}\right)^{-1}+y^{2} \Theta^{\sigma}\right),
\end{aligned}
$$

where $\mathbf{C}$ is a constant vector independent of $(x, y)$ which is bounded above by $M \delta_{*}^{4} \Theta^{\sigma}$. Also $|\mathbf{E}|$ is bounded independently of $x, y, Q$ and $\delta$. Thus,

$$
\mathbf{G}(x, y)=\mathbf{C}+L\left(\begin{array}{l}
x \\
y
\end{array}\right)+\mathbf{T}(x, y)
$$

where $L$ is a linear operator which, from a direct calculation, is seen to be invertible. If we define the operator $\mathbf{J}$ mapping $\mathbb{R}^{2}$ into itself by

$$
\mathbf{J}(x, y)=-\left(L^{-1} \mathbf{C}+L^{-1} \mathbf{T}(x, y)\right),
$$

then, provided that $Q_{*}$ is suitably large, a direct calculation shows that $\mathbf{J}$ maps the set $B$ into itself, where $B$ is the ball

$$
B=\left\{(x, y):\left(x^{2}+y^{2}\right)^{1 / 2} \leq \frac{4 \delta_{*}^{4} \Theta^{\sigma} M}{(p+1) \omega E \sqrt{a_{0}^{2}+b_{0}^{2}}}\right\} .
$$

We may therefore apply the Brouwer Fixed Point Theorem to conclude that $\mathbf{J}$ has a fixed point in $B$. This point $(x, y)$ satisfies both $\mathbf{G}(x, y)=$ 0 and

$$
\left(x^{2}+y^{2}\right)^{1 / 2} \leq A \delta_{*}^{4} \Theta^{\sigma},
$$

where $A$ is a constant independent of $\delta_{*}, Q_{*}$ and $\Theta$. By substituting for $Q$ and $\delta$, and then taking $\Theta$ to have the upper limiting value of $Q_{*}^{-\frac{\sigma}{(2-\sigma) \alpha}}$, we deduce the values given in (4.7) and (4.8). We have obtained a $C^{2}$ solution of (1.10) for each fixed $m$ large. Hence, we obtain the following theorem which implies Theorem 1.1.

Theorem 4.1. For any integer $m \gg 1$ large and $Q$ and $\delta$ given in (4.7) and (4.8), problem (4.1) admits a $C^{2}$ solution $w_{Q, \delta}(\theta)$. Moreover, there is $\Theta=\left|O\left(Q^{\frac{-\sigma}{(2-\sigma) \alpha}}\right)\right|$ such that

$$
\begin{aligned}
w_{Q}^{\text {inn }}(\Theta) & =w_{\delta}^{\text {out }}(\Theta), \\
\left(w_{Q}^{\text {inn }}\right)_{\theta}^{\prime}(\Theta) & =\left(w_{\delta}^{\text {out }}\right)_{\theta}^{\prime}(\Theta) .
\end{aligned}
$$

As a consequence, problem (1.10) admits infinitely many radially symmetric solutions. 


\section{REFERENCES}

[1] C. Budd and J. Norbury, Semilinear elliptic equations and supercritical growth, J. Differential Equations 68 (1987), 169-197.

[2] E.N. Dancer, Z.M. Guo and J.C. Wei, Non-radial singular solutions of LaneEmden equation in $\mathbb{R}^{N}$, Indiana Univ. Math. J. to appear.

[3] J. Davila and A.C. Ponce, Hausdorff dimension of ruptures sets and removable singularities, CRAS 346(2008), no.1-2, 27-32.

[4] Y.H. Du and Z.M. Guo, Positive solutions of an elliptic equation with negative exponent: stability and critical power, J. Differential Equations 246 (2009), 2387-2414.

[5] P. Esposito, Compactness of a nonlinear eigenvalue problem with a singular nonlinearity, Comm. Contemp. Math. 10 (2008), 17-45.

[6] P. Esposito, N. Ghoussoub and Y. Guo, Compactness along the branch of semistable and unstable solutions for an elliptic problem with a singular nonlinearity, Comm. Pure Appl. Math. 60 (2007), 1731-1768.

[7] P. Esposito, N. Ghoussoub and Y. Guo, Mathematical analysis of partial differential equations modelling electrostatic MEMS. Courant Lecture Notes in Mathematics 20. AMS, Providence, RI 2010.

[8] N. Ghoussoub and Y. Guo, On the partial differential equations of electrostatic MEMS devices: stationary case, SIAM J. Math. Anal. 38 (2006/07), 1423-1449.

[9] H.X. Guo, Z.M. Guo and K. Li, Positive solutions of a semilinear elliptic equation with singular nonlinearity, J. Math. Anal. Appl. 323 (2006), 344359.

[10] Z.M. Guo, D. Ye and F. Zhou, Existence of singular positive solutions for some semilinear elliptic equations, Pacific J. Math. 236 (2008), 57-71.

[11] Z.M. Guo and J.C. Wei, On the Cauchy problem for a reaction-diffusion equation with a singular nonlinearity, J. Differential Equations 240 (2007), 279-323.

[12] Z.M. Guo and J.C. Wei, Symmetry of non-negative solutions of a semilinear elliptic equation with singular nonlinearity, Proc. R. Soc. Edinb. 137A (2007), 963-994.

[13] Z.M. Guo and J. C. Wei, Asymptotic behavior of touch-down solutions and global bifurcations for an elliptic problem with a singular nonlinearity, Comm. Pure Appl. Anal. 7(2008), no.4, 765-786.

[14] D.D. Joseph and T.S. Lundgren, Quasilinear Dirichlet problems driven by positive sources, Arch. Rational Mech. Anal. 49 (1973), 241-269.

[15] H. Q. Jiang and W.M. Ni, On steady states of van der Waals force driven thin film equations, European J. Appl. Math. 18 (2007), 153-180.

[16] L. Ma and J.C. Wei, Properties of positive solutions to an elliptic equation with negative exponent, J. Funct. Anal. 254(2008), no.4, 1058-1087. 
Department of Mathematics, Henan Normal University, XinXiang, 453007, CHINA

E-mail address: gzm@htu.cn

Department of Mathematics, The Chinese University of Hong Kong, Shatin, Hong Kong

E-mail address: wei@math.cuhk.edu.hk 